\title{
Physiologically-Regulated Expression Vectors for Gene Therapy
}

\author{
Olivia Hibbitt and Richard Wade-Martins \\ University of Oxford, Department of Physiology Anatomy and Genetics \\ United Kingdom
}

\section{Introduction}

Gene-replacement gene therapy has been under development for a number of years. In spite of the large amount of research invested into developing gene therapy for the treatment of recessive genetic disorders only a limited number of patients world-wide have received the benefits. In addition, several high profile adverse events in gene therapy trials have lead to an increasing awareness of the challenges facing gene therapy treatments before they become established in the clinic. This has necessitated the development of novel advances in gene therapy vector design and delivery. This chapter will focus on the development of gene expression vectors incorporating native genomic regulatory elements that ensure transgene expression is physiologically relevant. Three main advances will be discussed here in detail; the use of whole genomic DNA loci to ensure physiologically-regulated transgene expression; development of viral vectors based on the herpes simplex virus type 1 for delivery of whole genomic DNA loci; and the development of genomic mini-gene vectors that contain native regulatory regions for the physiologically-regulated expression of cDNA mini-genes.

The principal aim of gene-replacement gene therapy is to complement the loss of function of an endogenous gene by supplying an exogenous 'working' copy in trans. The conventional approach to this is to supply a wild-type cDNA copy of the gene in a small vector in which transgene expression is controlled by a strong heterologous promoter, such as the immediate early promoter of cytomegalovirus (pCMV). The advantage of this approach is that the vectors are easy to use, have high levels of transgene expression, and fit easily into most viral delivery systems such as lentivirus and adenovirus. However, expression from these vectors is characteristically short-term and wide-spread with no tissue specificity or temporal regulation. One alternative to heterologous expression vectors for gene therapy is to utilise native genomic DNA regulatory elements to ensure gene expression that is both spatially and temporally regulated. A highly effective means of achieving gene expression that is physiologically-regulated is through the use of whole genomic loci which contain all introns, exons and regulatory regions in the correct genomic context. Expression from whole genomic loci has been proven to recapitulate endogenous expression. In the context of gene therapy, delivery of whole genomic loci using bacterial artificial chromosomes (BAC) has been shown to be an effective means of complementing gene deficiencies. Delivery of BAC vectors carrying complete loci encoding, for example, the genes for the human low density lipoprotein receptor $(L D L R)$, the Friedreich's ataxia (FRDA) frataxin protein (FXN), 
microtubule associated protein tau (MAPT) and hypoxanthine phosphoribosyltransferase (HPRT) effectively rescue gene deficiencies in vitro. It has also been shown that the complementation of gene deficiency is responsive to changes in the cellular milieu, an important point for conditions where gene expression is controlled by cellular signalling pathways and where over-expression of the gene is toxic.

Historically, BAC vectors have been discounted for gene therapy purposes as there was no viral delivery system with the transgene capacity for a whole genomic locus which may be $\geq 100 \mathrm{~kb}$. Recently, viral vectors based on the herpes simplex virus type (HSV-1) amplicons have been developed and shown to have a transgene capacity of over $100 \mathrm{~kb}$ and a broad cell tropism making them an attractive means of delivering large transgenes for the purposes of gene therapy. Currently HSV-1 amplicons have been used in a number of gene complementation studies which will be reviewed in detail here.

Recent work in our laboratory has adapted the genomic locus approach for the treatment of familial hypercholesterolaemia (FH). FH is a condition caused by mutations in the LDLR gene and represents a unique challenge in gene therapy. Over-expression of the LDL receptor leads to a toxic accumulation of intracellular cholesterol. It is therefore essential that $L D L R$ transgene expression is appropriately regulated. We have generated gene expression vectors in which expression of the LDLR cDNA was controlled by $10 \mathrm{~kb}$ of genomic DNA encompassing key regulatory regions in the $L D L R$ genomic DNA promoter. These regulatory regions sense the levels of intracellular cholesterol. When cholesterol levels in the cell are high, LDLR expression is low; when the cholesterol stores become depleted, expression of the LDL receptor is high. Delivery of the LDLR mini-gene expression vectors in vitro and in vivo lead to efficient and prolonged $L D L R$ gene expression in vivo that is sensitive to changes in cellular cholesterol and resulted in a decrease in circulating cholesterol in receptor deficient mice.

\section{Physiologically-relevant gene expression vectors: use of a complete genomic locus}

The central aim of gene-replacement gene therapy is to complement the loss of function of an endogenous gene by supplying a working copy of that gene in trans. Classically this was achieved using cDNA coding for the endogenous gene under the expression control of constitutively active exogenous promoters such as the immediate early promoter from the cytomegalovirus (pCMV). In general transgene expression levels from these vectors are characteristically high and therefore therapeutic effect is seen in some conditions. Clinical trials have demonstrated that these vectors are effective in ameliorating the symptoms of conditions such as haemophilia (Manno et al. 2006) and several severe combined immunodeficiencies (SCID) such as X-linked SCID (Cavazzana-Calvo et al. 2000), adenosine deaminase deficiency (Aiuti et al. 2009) and chronic granulomatous disease (Ott et al. 2006). The SCID trials also demonstrated that delivery of transgenes in the context of small cDNA vectors with no native expression control did have a number of issues including cell transformation.

\subsection{Complete genomic locus ensures gene expression in the correct genomic context}

There are several issues which may confound the use of cDNA expression cassettes to complement the loss of function of an endogenous gene: aberrant spatial expression dynamics resulting in gene expression in 'off-target' cells; aberrant temporal dynamics 
resulting in continuous expression of a transgene with possible cytotoxic consequences; transgene over-expression at supra-physiological levels; and, the inability to produce multiple splice variants.

Transgenic mice offer an interesting insight into the benefits of using native genomic loci over cDNA expression systems to investigate the function of genes. For some genes it is essential that they are expressed in the correct spatial, developmental and temporal context to ensure functionality. The $\beta$-globin gene cluster is an excellent example of this. This genomic locus consists of five separate genes $\left(5^{\prime}-\varepsilon-{ }_{-}^{-} \gamma^{-A} \gamma^{-}-\delta-\beta-3^{\prime}\right)$ that are expressed at different developmental stages (Huang et al. 2000). The $\gamma$ genes are expressed in foetal erythroid tissues while the $\delta$ and $\beta$ are expressed in adult haematopoietic cells of the erythroid lineage. The expression of these genes in under the control of a region called the locus control region (Huang, Liu et al. 2000). In mice expressing the $\beta$-globin from a cDNA expression plasmid without the locus control region, low levels of protein are detected with no tissue-specificity (Magram et al. 1985; May et al. 2000; Vadolas et al. 2005). The use of the entire genomic locus of the $\beta$-globin gene cluster which included the locus control regions resulted in spatial and temporal expression profiles that mimicked the native profile (Porcu et al. 1997; Vadolas, Wardan et al. 2005).

Further examples of the advantages of using the complete genomic locus comes from mice lacking either the frataxin (Fxn) gene (Cossee et al. 2000; Miranda et al. 2002) or the cystic fibrosis transmembrane conductance regulator (Cftr) gene (Zhou et al. 1994; Manson et al. 1997), mouse models of Friedrich's ataxia and cystic fibrosis, respectively. Mice lacking frataxin die at embryonic day six and crossing heterozygous knock-out mice with mice expressing the full genomic locus of the human FXN gene from a bacterial artificial chromosome (BAC) rescues the phenotype and expression patterns of mRNA and protein was physiological (Sarsero et al. 2004). Transgenic mice lacking the endogenous Cftr gene but expressing the full $200 \mathrm{~kb}$ of the human CFTR gene in a yeast artificial chromosome (YAC) show correct expression of CFTR protein in the appropriate spatial and temporal context (Manson, Trezise et al. 1997).

Other examples include comparisons between mice expressing the amyloid precursor protein (APP) as either a cDNA construct or as a complete locus within a YAC. The APP gene is involved with the development of Alzheimer's disease. It is a complex genomic locus comprising 18 exons that are alternatively spliced to give rise to four distinct transcripts (Hsiao et al. 1996). Mice expressing the APP cDNA vector do not express APP protein in the correct genomic context limiting the relevance of biological information obtained from these animals (Lamb 1995; Lamb et al. 1997). Mice expressing APP from the YAC construct displayed physiologically-relevant APP protein expression making them a far superior tool for the study of how APP might contribute to the development of Alzheimer's disease (Lamb, Call et al. 1997).

The advantages of using BAC plasmids to generate transgenic mice is now widely accepted. BAC transgenics have been shown, for example, to rescue knockout phenotypes in mice lacking the Pkd1 gene involved in polycystic kidney disease (Pritchard et al. 2000) and mice lacking $\beta$-globin genes (Vadolas et al. 2002; Jamsai et al. 2005; Vadolas, Wardan et al. 2005; Jamsai et al. 2006). BACs have also been useful in investigating novel genomic expression control regions. A negative regulatory region in the Wilson's disease gene was characterised using BAC plasmids (Bochukova et al. 2003). BACs were also used to characterise the locus control regions responsible for the differential expression of Myf5 in skeletal muscle (Carvajal et al. 2001; Zammit et al. 2004). In addition to this, insertion of reporter genes into 
BAC plasmids has enabled the understanding of spatial and temporal expression dynamics of many genes such as Nkx2-5 (Chi et al. 2003). Recently BAC transgenesis has been used in studies of immunomodulation (Kulik et al. 2011), blood vessel development (Ishitobi et al. 2010) and in generating mouse models of Parkinson's disease that more closely recapitulate deficits in the human disease ( $\mathrm{Li}$ et al. 2009). These studies represent a small sub-section of the work being performed using whole genomic loci to better understand gene function. They demonstrate that the use of native regulatory regions can yield more biologicallyrelevant data than over-expression studies. This is important in the generation of mouse models of disease and also in the development of therapeutic protocols to treat genetic disease.

\subsection{Complete genomic locus for therapy}

Transgenic animals offer extensive evidence that the use of cDNA expression vectors often does not result in physiologically-relevant expression patterns. In terms of gene therapy the use of these cDNA vectors may not be appropriate for diseases where the correct physiological expression of the transgene is vital for therapeutic effect and to protect cells from ectopic or cytotoxic over-expression, where proteins expressed with no control result in pathological changes in the transduced cell.

The use of a complete genomic DNA region in the design of gene therapy vectors is still a relatively new field. Manipulation and use of such large pieces of DNA can be challenging. Success has been seen however with a range of genes using a number of different techniques to isolate and deliver the locus. In vitro studies demonstrated that it was possible to achieve gene expression following non-viral BAC plasmid delivery. The gene involved in LeshNyan syndrome, hypoxanthine phosphoriribosyltransferase (HPRT) was delivered to HPRT deficient fibroblasts and resulted in sustained physiological levels of HPRT (Wade-Martins et al. 2000). Lipofection and an integrin targeting peptide were used to deliver a $143 \mathrm{~kb}$ BAC encompassing the locus of Nijmegen breakage syndrome gene (NBS1) resulting in expression of the NBS1 gene product, nibrin (White et al. 2003). BACs have also been generated that contain the CFTR locus (Kotzamanis et al. 2009) delivery of which to CMT-93 cells resulted in mRNA expression that was correctly spliced (Kotzamanis, Abdulrazzak et al. 2009).

In vivo non-viral delivery of plasmids containing large genomic DNA inserts has been achieved in two studies. In the first study hydrodynamic tail vein injection or lipofection was used to successfully deliver plasmids containing $150 \mathrm{~kb}$ of non-gene specific DNA (Magin-Lachmann et al. 2004). We have also demonstrated efficient delivery of a $135 \mathrm{~kb}$ genomic insert containing the full human low density lipoprotein receptor (LDLR) genomic locus for the treatment of Familial Hypercholesterolaemia (FH). We showed that up to 4 months following hydrodynamic tail vein injection, human LDLR protein was detectible in the livers of recipient mice (Hibbitt et al. 2007).

Alternatives to BAC plasmids for delivery of large genomic inserts are also being investigated. Human artificial chromosomes (HACs) for example offer advantages over the bacterial counterparts. HAC vectors are able to replicate and segregate without integration into the host-cell chromosomes and are capable of carrying very large amounts of DNA. HACs have been shown to be an effective means of generating transgenic mice (Suzuki 2006). They have also been used to express HPRT (Moralli et al. 2006) and CFTR complete genes (Rocchi et al. 2010). In addition a HAC containing the entire $2.4 \mathrm{Mb}$ genomic locus of the human dytrophin gene was used to stably maintain expression of human dystrophin in 
mouse embryonic stem cells (Hoyshiya 2009) without any integration into the host cell chromosomes. These cells were used to generate chimeric mice analysis of which showed correct tissue-specific dystrophin expression. As the HACs are stable they could be useful in cell therapies in the future.

Viral vectors have also been developed to achieve infectious delivery of large genomic sequences. A gutless adenovirus with a transgene capacity of $36 \mathrm{~kb}$ was used to deliver the whole locus of the human a1 antitrypsin gene (SERPINA1) to mice (Schiedner et al. 1998). Vectors based on the Epstein Barr virus have also been used to deliver $120 \mathrm{~kb}$ of genomic DNA to cells of lymphoblast and B-cell lineage (White et al. 2002). Other viral systems have been under investigation, such as $\mathrm{CMV}$ which has a very large transgene capacity of around $210 \mathrm{~kb}$ and a strong haematopoietic cell tropism (Borst and Messerle 2000; Borst and Messerle 2003). However, the best characterised is amplicon vectors based on Herpes Simplex 1 (HSV-1).

\subsection{Infectious delivery of a complete genomic locus - HSV-1 amplicons}

The large size of a compete genomic locus precludes their use with most viral vector systems which typically have a transgene capacity of less than $20 \mathrm{~kb}$. Vectors based on the herpes virus family however have a much larger transgene capacity. HSV-1 in particular is well described and widely used. Wild-type HSV-1 infects mucosa and establishes a latent phase in sensory neurons. HSV-1 infection produces cold sores in symptomatic infected individuals and $90 \%$ of the population has circulating antibodies (Corey and Spear 1986; Bowers et al. 2003). The HSV-1 genome consists of $152 \mathrm{~kb}$ of double stranded DNA. Of this only two non-coding regions are required for the packaging of DNA plasmids into HSV-1 virions (Spaete and Frenkel 1982; Spaete and Frenkel 1985). Inclusion of these two packaging signals, the OriS and pac, into DNA plasmids will promote their packaging into HSV-1 virions in the presence of the HSV-1 genome in trans. Plasmids are packaged head to tail in concatemers up to $150 \mathrm{~kb}$. The average size of a human genomic locus is around $40 \mathrm{~kb}$ and the $150 \mathrm{~kb}$ capacity of HSV-1 amplicons potentially allows delivery of up to $90 \%$ of genomic loci as infectious particles, making the vector a highly versatile viral packaging system (Senior and Wade-Martins 2005; Hibbitt and Wade-Martins 2006).

HSV-1 amplicons are capable of infecting dividing and non-dividing cells including, but not limited to; neurons, such as those of the dorsal root ganglion (Marsh et al. 2000), thalamus (Costantini et al. 1999), cortex (Agudo et al. 2002), hippocampus (Adrover et al. 2003), glial cells (Marsh, Dekaban et al. 2000), gliomas (Shah et al. 2004), skeletal muscle (Wang et al. 2000; Wang et al. 2002) and osteoblasts (Xing et al. 2004). HSV-1 amplicons also retain their ability for retrograde transport in neuronal axons allowing for the possibility of peripheral delivery for centrally located targets. For example inoculation of the foot pad in diabetic rats with HSV-1 amplicons expressing nerve growth factor (NGF) resulted in NGF expression in the dorsal root ganglion and protected against diabetes associated peripheral neuropathy (Goss et al. 2002).

One of the key concerns with any viral vector system is safety. HSV-1 amplicons are nonintegrating viruses which thus avoids issues of cell transformation by insertional mutagenesis. The packaging of OriS and pac containing plasmids into HSV-1 amplicons requires the presence of the HSV-1 genome in trans. To improve vector safety helper virusfree HSV-1 amplicon packaging systems have been developed. The supply of the HSV-1 genome in trans has been achieved by the use of BAC plasmids encompassing the full genome and lacking the packaging signal (Saeki et al. 1998; Stavropoulos and Strathdee 
1998). Further safety mechanisms were then added to the system. One recent packaging BAC now widely used has both the packaging signal (pac) and the essential gene, ICP27, deleted, and is also oversized to preclude its inclusion into HSV-1 virions (Saeki et al. 2003). It has also been demonstrated that this packaging system is an efficient means of generating infectious BAC (iBAC) particles that have been successfully used for gene expression in vivo and in vitro. Overall, helper virus-free packaging systems for HSV-1 amplicons result in vector stocks with a much reduced immune-response in vivo (Olschowka et al. 2003).

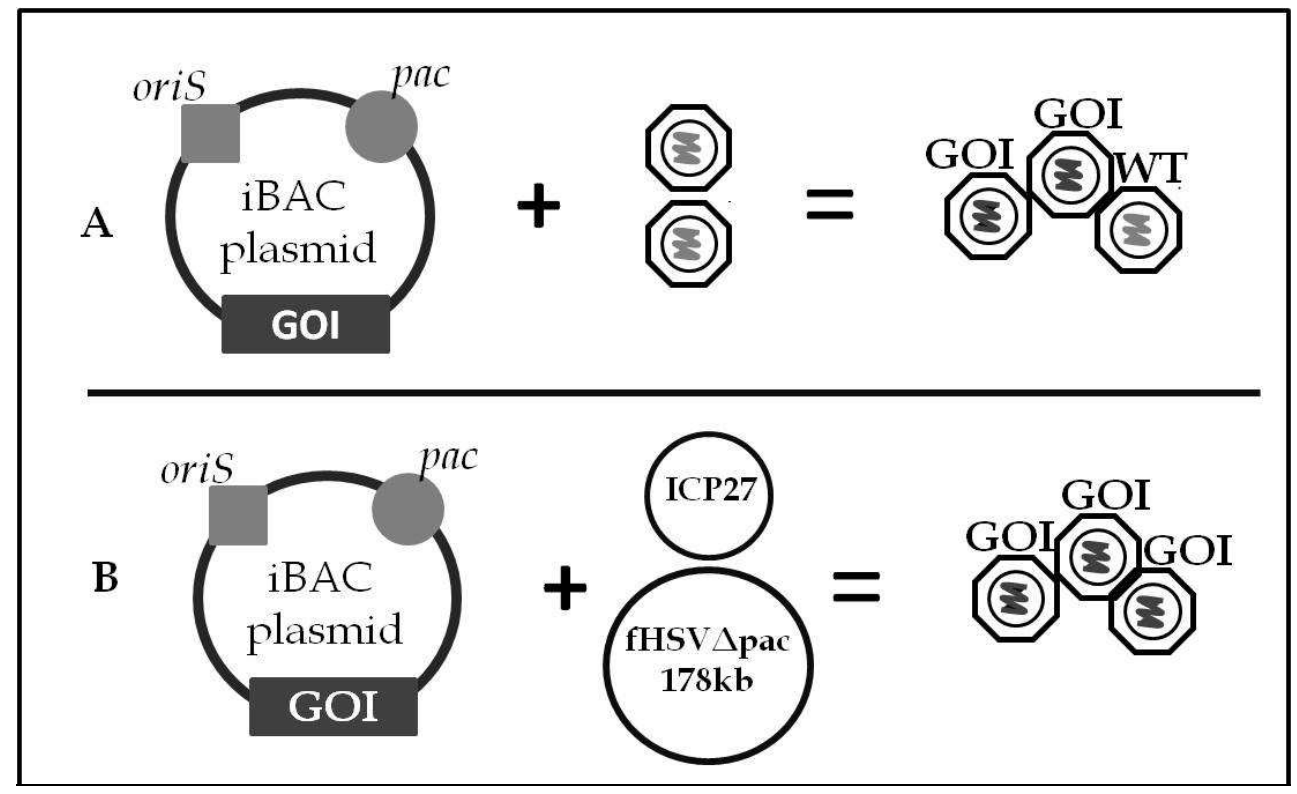

Fig. 1. Packaging of HSV-1 amplicons.

Schematic showing packaging of OriS and pac containing plasmids into HSV-1 virions. A) AniBAC plasmid containing the gene of interst (GOI) is packaged using packaging virus to supply the HSV-1 genome in trans. This results in viral stocks that contain virions only carrying the iBAC GOI plasmid and wild-type-like virus. B) An improved packaging system using two plasmids in place of the wild-type-like virus. An oversized, ICP27 deleted BAC plasmid and a small plasmid that contains ICP27. This results in viral stocks that only contain virions with GOI containing iBAC.

HSV-1 delivers DNA to the cell as an extrachromosomal element and hybrid vectors have been designed to promote persistence of episomal vector DNA (Figure 2). The best described of these is the HSV-1/EBV hybrid vectors. The inclusion of the EBV latent origin of replication OriP and the EBV nuclear antigen (EBNA-1) promotes replication and segregation of DNA during cell division (Wang and Vos 1996; Wade-Martins et al. 2001; Wade-Martins et al. 2003; Muller et al. 2005). Mammalian-based systems have also been investigated. The inclusion of scaffold matrix attachment regions (SMARs) to an iBAC containing the human LDLR gene resulted in the establishment of stable, episomal LDLR expression in cell lines (Lufino et al. 2007). Successful delivery and establishment of HACs has been shown in cells transduced with iBAC vectors carrying alpha satellite DNA inserts (Moralli, Simpson et al. 2006). 


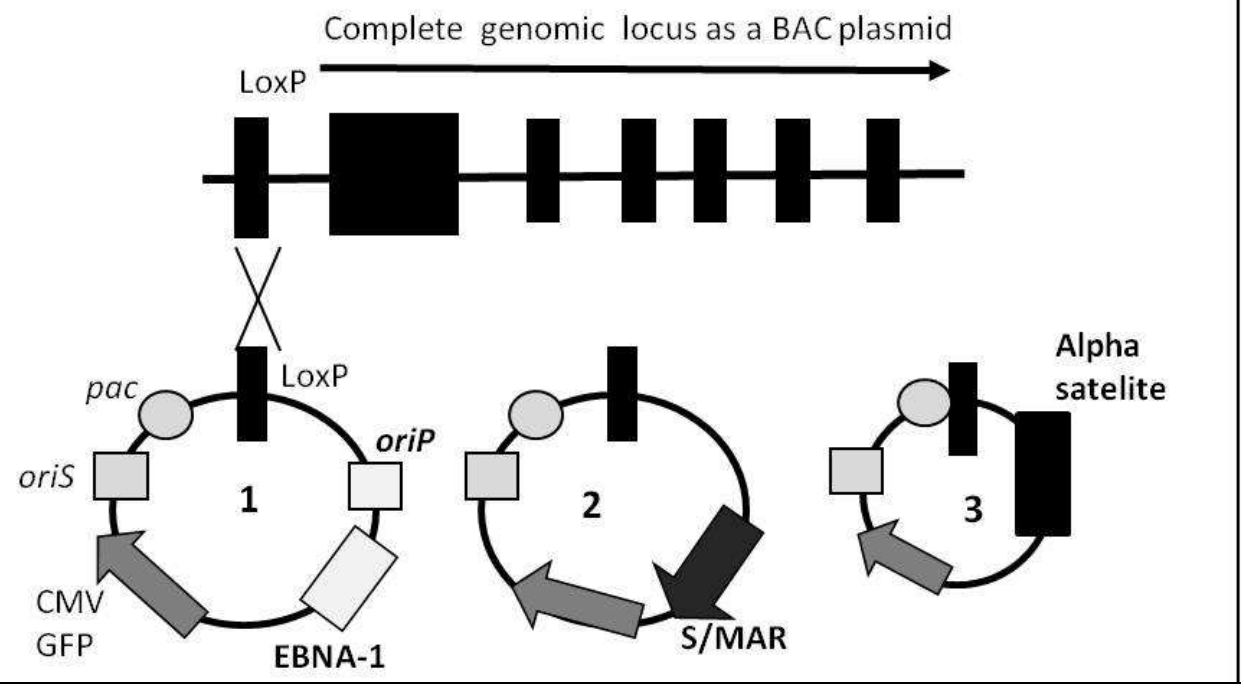

A BAC plasmid containing complete genomic locus can be retrofitted using cre-LoxP recombination with small plasmids containing elements essential for extrachromosomal maintance of delivered plasmids. Suggested in this figure is the incorpation of a CMV-green fluorescence protein (CMVGFP) reporter gene cassette to allow for assessment of delivery. The HSV-1 packaging signals (pac and oriS) and retention elements. ; 1) Contains the episomal retention elements from the Epstein Barr virus (EBNA-1 and oriP), 2) contains the S/MAR retention elements, 3) Alpha satelite DNA promotes the generation of human artificial chromosomes following delivery.

Fig. 2. Extrachromosomal retention elements.

The use of iBAC vectors in gene therapy is still evolving and a number of studies have demonstrated that these vectors are capable of efficient delivery and genetic complementation. Recent work has used the delivery and expression of the complete genomic of two genes key to the development of Alzheimer's disease and Parkinson's disease, microtubule associated protein tau (MAPT) and alpha synuclein (SNCA) to study gene function. Amplicon iBAC vectors carrying the $143 \mathrm{~kb} M A P T$ locus or the $135 \mathrm{~kb} S N C A$ locus were used to infect cellular models of neurodegeneration (Peruzzi et al. 2009). Expression of MAPT and SNCA in cells infected with the iBAC vectors was similar to endogenous human levels. It was found by comparing transgene expression in primary neuronal and glial cultures that expression from the MAPT locus was strictly regulated by developmental time-point and cell type. Multiple transcripts were observed which mimicked the expression pattern seen in humans. Infection of MAPT-deficient neurons in culture with the $M A P T$ iBAC vector rescued the cellular phenotype, restoring the normal response to $A \beta$-peptide (Peruzzi, Lawler et al. 2009). This delivery system provides an effective means of investigating neurodegeneration in cell models.

Success had already been seen previously using iBAC vectors coding for the HPRT (WadeMartins, Smith et al. 2001) as described in Section 2.2 or bone morphogenic protein 2 (BMP2) (Xing, Baylink et al. 2004) loci. The role of BMP2 in osteoblast formation was investigated using iBAC delivery of the complete BMP2 locus (Xing, Baylink et al. 2004). BMP2 has been 
implicated in the differentiation of osteoblasts by altering alkaline phosphatase activity in precursor cells (Thies et al. 1992). When the iBAC-BMP2 vector was used to infect a preosteoblast cell line differentiation was observed consistent with appropriate BMP2 expression (Xing, Baylink et al. 2004).

Small cDNA-based vectors are not suitable to express loci which undergo complex splicing, such as the MAPT locus discussed above. Another example is the human CDKN2 genomic locus, an especially complex region. Expression from the CDKN2 locus results in five different genes from only six exons (Sharpless and DePinho 1999). Two of the genes $p 16^{I N K 4 a}$ and $p 14 A R F$ are particularly complex. Two separate promoter regions control expression of each gene. Separate first exons are spliced with second and third exons which are shared between the two transcripts (Sharpless and DePinho 1999). Both of these genes are involved in cell cycle control. They are being investigated as potential targets for cancer gene therapy. The $132 \mathrm{~kb} C D K N 2$ locus was delivered as a iBAC vector to CDKN2 knockout glioma cells. Cells transduced with the iBAC vector has physiological levels of expression and correct splicing of three gene products of the CDKN2 locus, $p 15, p 16^{I N K 4 a}$ and $p 14^{A R F}$ (Inoue et al. 2004). Cells also displayed a phenotype of reduced growth consistent with expression of cell cycle arrest genes (Inoue, Moghaddam et al. 2004).

HSV-1 amplicons have been used to deliver the complete genomic locus of the LDLR gene to cell models of FH (Wade-Martins, Saeki et al. 2003; Lufino et al. 2007; Lufino, Manservigi et al. 2007). It was shown that delivery of the $L D L R$ locus as an iBAC to $L d l r$ deficient CHO cells and $\mathrm{FH}$ patient fibroblasts lead to attenuation of the cellular phenotype as seen by normalisation of uptake and internalisation of LDL (Wade-Martins, Saeki et al. 2003; Lufino, Manservigi et al. 2007). Long-term expression was achieved by the use of the EBV episomal retention elements (Wade-Martins, Saeki et al. 2003) or scaffold matrix attached regions (Lufino, Manservigi et al. 2007). Both episomal retention systems promoted efficient establishment of the LDLR locus as a extrachromosomal element (Wade-Martins, Saeki et al. 2003; Lufino, Manservigi et al. 2007). Expression from this locus was down-regulated by sterol treatment through interaction with response elements in the promoter region (WadeMartins, Saeki et al. 2003; Lufino, Manservigi et al. 2007).

The first example of in vivo infectious delivery of a whole genomic locus using the iBAC delivery system was recently described (Gimenez-Cassina et al. 2011). The entire $135 \mathrm{~kb}$ FXN gene was delivered as an iBAC directly to mouse cerebellum using intracranial injection. Analysis of expression of the LacZ reporter gene expressed from within the FXN locus showed large numbers of transduced cells in the cerebellum up to 75 days postinjection. This was also compared to reporter gene expression from the viral HSV-1 IE4/5 promoter which was short-lived in vivo from the same vector. This work is an elegant demonstration of the potential of BAC plasmid delivery in vivo to result in high-level sustained transgene expression.

The LDLR expression and FXN iBAC delivery studies represent an interesting proof of principle in the use of complete genomic regions to treat genetic disease via gene therapy. Delivery of the whole genomic locus is an elegant way to provide the therapeutic gene in its correct genomic context, ensuring that complementation can be physiologically-relevant, cell-specific and temporally-regulated. This has the potential effect of decreasing genotoxicity and improving the safety and efficacy of gene therapy vectors. However, the use of large genomic inserts can be technically challenging. Work is underway to develop gene expression vectors that combine the gene-regulation of a large insert with the convenience of a small mini-gene vector. 


\section{Physiologically-relevant gene expression vectors: use of native regulatory regions}

Whole genomic loci represent an excellent means of ensuring physiologically-relevant expression in target cells. However, the large size of BAC plasmids precludes their use in all but a few viral vector systems. Although, non-viral systems such as hydrodynamic tail vein injection offer excellent means of delivery to target certain tissues, for many applications BAC-sized plasmids may not be practical. Many studies have attempted to combine the advantages of cDNA vectors (small size, high transduction or transfection efficiency, and high levels of protein expression) with an advantage of a whole genomic locus, being regulated physiologically-relevant expression. Depending on the gene of interest it may be necessary only to ensure expression of the transgene is restricted to a particular cell type; alternatively, it may be necessary to ensure transgene expression also tracks changes in cell physiology to ensure therapeutic and not pathologic transgene expression.

There has been extensive research into targeting gene expression to desired tissues using transcriptional restriction. Such work uses well-characterised promoters and enhancer regions that limit transgene expression to certain desired cell types where they are active. Liver-directed gene expression for example has been achieved using the promoter regions from either the albumin (Follenzi et al. 2002) or a1 antitrypsin genes (Le et al. 1997) to target expression of clotting factors to the liver to treat the haemophilia family of diseases. Targeting gene expression to cells in vascular wall is possible using endothelial cell restricted expression through the use of promoter such as VE-cadherin or VEGFR-1 (Quinn et al. 2000; Nicklin et al. 2001). Vascular smooth muscle specific expression has been achieved using promoters like the SM22 promoter (Imai et al. 2001) and was successfully used to target expression of heme oxygenase 1 to the vascular endothelium.

For some diseases it is not enough to limit expression to cell type. The temporal dynamics of gene expression is also important. One novel way of achieving physiological expression using small cDNA vectors is to generate a genomic mini-gene construct that uses native gene expression elements with a cDNA transgene. Wiskott-Aldrich syndrome (WAS) is an excellent example of the need for native regulatory elements to ensure correct expression dynamics. WAS is an X-linked recessive disease caused by mutations in the WAS protein gene (WAS) and defined by thrombocytopenia. WAS is expressed in haematopoietic cells at different concentrations depending on the cell type (Toscano et al. 2008). Over-expression in non-haematopoietic cells has been shown to be cytotoxic (Toscano, Frecha et al. 2008). Lentiviral delivery of WAS cDNA vectors where expression is governed by different fragments of the WAS gene promoter ranging from $500 \mathrm{bp}$ to $1600 \mathrm{bp}$ has demonstrated restricted expression of WASP in haematopoietic cells (Dupre et al. 2004; Martin et al. 2005; Leuci et al. 2009). These vectors were also able to correct the genetic defect in Was knockout mice and transduce haematopoietic cells from WAS patients. However, these relatively small promoter fragments were unable to achieve complete physiological regulation and some target cell types did not exhibit correct transgene expression profiles (Frecha et al. 2008). This may be because the promoter fragment was too small to contain all necessary machinery for all target cells.

One example in which clinical success has been seen with a vector containing native regulatory elements is in treatment of Leber's congenital amaurosis, a group of recessive congenital rod-cone dystrophies. Mutations in a retinal pigment epithelium specific gene called RPE65 causes impaired vision from birth that degenerates to complete blindness later 
in life. An adeno-associated virus was constructed that contained the RPE65 cDNA under the expression control of 1600 bp human RPE65 promoter (Le Meur et al. 2007). The use of the native promoter region of the RPE65 gene effectively targeted expression to the retinal epithelium. This was shown to be effective at improving vision in a naturally occurring animal model; the Swedish Briard dog. This vector is now in clinical trials to treat this condition in humans (Bainbridge et al. 2008).

These two examples use only a minimal promoter region, which may be appropriate for those genes where regulatory elements are located in a small region proximal to the start of the coding region. However, as was seen in the WAS example, a larger portion of genomic DNA may be necessary for full physiological regulation. In our laboratory we have investigated the use of a $10 \mathrm{~kb}$ piece of genomic DNA to ensure fully physiological expression of the low density lipoprotein receptor gene (LDLR) for functional complementation in vitro and in vivo for the treatment of familial hypercholesterolaemia $(\mathrm{FH})$. FH is caused by mutations in the LDL receptor which binds and internalises LDL cholesterol in response to low intracellular cholesterol levels. Gene therapy for FH been under investigation for a number of years with many published investigations reporting lowering of plasma cholesterol following delivery of cDNA vectors where expression is driven by heterologous promoters and delivery is achieved by virus-mediated liver-directed transduction with retrovirus (Miyanohara et al. 1988; Wilson et al. 1988; Chowdhury et al. 1991; Grossman et al. 1995; Kankkonen et al. 2004), adenovirus (Ishibashi et al. 1993; Kozarsky et al. 1994; Li et al. 1995; Kozarsky et al. 1996; Nomura et al. 2004; Jacobs et al. 2008; Van Craeyveld et al. 2011) and adeno-associated virus (Lebherz et al. 2004; Kassim et al. 2010). These previous studies included a clinical trial (Grossman, Rader et al. 1995) that showed no evidence of long-term therapeutic effect.

FH represents a significant challenge for gene therapy due to the regulation of $L D L R$. There are three issues that need to be overcome by a therapeutic protocol. Firstly, cholesterol biosynthesis in the liver is constitutive. This means any gene therapy protocol needs to supply an agent that is not only capable of clearing cholesterol already present in the serum, it needs to clear all future cholesterol that will be synthesised by the liver. This puts huge demands on the transduced cells. The second issue is that the LDLR locus is tightly regulated by a negative feedback system. Expression from the $L D L R$ genomic locus is controlled by levels of intracellular cholesterol. When intracellular levels of cholesterol fall $L D L R$ expression is triggered by the binding of sterol response element binding proteins (SREBP) to the sterol response elements in the promoter region (Sudhof et al. 1987; Briggs et al. 1993; Horton et al. 2002). This drives expression of the LDL receptor which binds and internalises LDL particles from the circulation. As cellular cholesterol stores become replete the SREBP become less active and expression from the LDLR locus is repressed. There is evidence that suggests hepatocytes are only metabolically able to deal with a certain amount of cholesterol influx. Delivery of cDNA vectors with strong viral promoters driving $L D L R$ expression into cells, and animals, using adenovirus results in immediate, dramatic lowering of cholesterol. The initial lowering of cholesterol is slowly eroded over time as intracellular cytotoxic accumulation of cholesterol leads to apoptotic cell death and loss of the transduced population of cells. The third confounding factor is the physiological expression itself. It is apparent that physiologically-relevant expression of the LDLR is important for the function of the hepatocytes and the nature of the expression plasmid may limit its ability to clear large amounts of cholesterol from the blood. As the cholesterol stores 
in transduced cells become replete the expression of the LDLR transgene will be repressed effectively 'switching off' that particular cell's cholesterol processing ability, this could reduce the therapeutic effectiveness of physiological vectors.

We have previously shown that an iBAC vector containing the $135 \mathrm{~kb} L D L R$ genomic locus encompassing all 18 exons, intervening introns, sterol response elements and other regulatory elements all in the correct genomic context was capable of rescuing the genetic defect in cell models of FH (Wade-Martins, Saeki et al. 2003; Lufino, Manservigi et al. 2007). When we moved the focus of the work into animal models of $\mathrm{FH}$ we found that non-viral gene transfer via hydrodynamic tail vein injection to be an efficient means of vector delivery. Delivery of the LDLR BAC using hydrodynamic tail vein injection resulted in longterm expression of human LDLR in the liver over the full four month course of the experiment (Hibbitt, Harbottle et al. 2007). However, the level of hepatocyte transduction was insufficient to result in therapeutic lowering of cholesterol.

The challenge was to maintain physiologically-regulated expression while improving transfection efficiency using hydrodynamic tail vein injection. We built genomic DNA minigene vectors that contained $10 \mathrm{~kb}$ of genomic DNA encompassing the full genomic DNA promoter of the human LDLR gene (Hibbitt et al. 2010). This consisted of the 5 ' untranslated region, three sterol response elements, the transcription initiation sequence and eight $\mathrm{kb}$ upstream of genomic DNA that may contain as yet undescribed enhancer elements (Figure 3a). This genomic DNA promoter region was used to drive the cDNA of either the luciferase or human $L D L R$ genes. We have shown in vitro that the $10 \mathrm{~kb} L D L R$ promoter provides stable, long-term, physiological expression and provides functional complementation in cell culture in Ldlr deficient $\mathrm{CHO}$ cells and $\mathrm{FH}$ patient fibroblasts (Figure 3b). Physiological $L D L R$ promoter induction was demonstrated using either luciferase expression, or specific LDL binding and internalisation assays in the presence of modifiers of receptor expression, sterols or statins. Statins are specific inhibitors of the de novo cholesterol synthesis pathway. They act on the LDL receptor by decreasing the amount of cholesterol in the cell thereby upregulating expression from the $L D L R$ promoter. Incubation of cells with statins lead to a five-fold up-regulation of expression from the $10 \mathrm{~kb}$ promoter element. Sterols downregulate LDL receptor expression through association with sterol response elements in the $L D L R$ promoter region. Incubation with sterols lead to a $50 \%$ down-regulation of expression from the $10 \mathrm{~kb}$ promoter element.

Liver-directed delivery of LDLR mini-gene vectors in vivo using hydrodynamic tail vein injection resulted in expression from the $L D L R$ promoter element that was sensitive to drug administration in vivo. Pravastatin administration resulted in a five-fold increase in luciferase expression five days after delivery (Figure 3c). The inclusion of EBV episomal retention elements ensured long-term expression up to 240 cell cycles in vitro and 9 months in vivo (Hibbitt, McNeil et al. 2010).

This work describes the successful combination of genomic DNA regulatory elements with a mini-gene cDNA vector. Expression from this vector is physiologically-regulated by intracellular cholesterol levels. Delivery of the smaller-sized mini-gene vector is more efficient than with the full BAC and highlights the possibility of combining gene replacement gene therapy with traditional medical treatments. Combining gene delivery with treatment that will reduce the amount of cholesterol being synthesised by the liver could increase the power of the gene delivery ensuring efficient binding and internalisation of LDL to reduce plasma cholesterol levels. We have demonstrated in vivo that treatment with statin drugs increases the activity of the $L D L R$ promoter. We have also investigated the 
feasibility of using a more targeted approach. Statins work by inhibiting the conversion of HMG CoA to mevalonate by 3-hydroxy-3-methylglutaryl Coenzyme A reductase (HMGCR), the rate limiting step in cholesterol synthesis inhibitors. We have investigated whether knock-down of HMGCR might have a more specific effect leading to a greater reduction in cholesterol synthesis and therefore greater induction of LDLR. We have demonstrated that treatment with HMGCR-specific small-interferring RNAs and synthetic microRNAs leads to a ten-fold induction of the LDLR promoter in vitro and in vivo which resulted in a greater reduction in circulating LDL cholesterol in Ldlr knockout mice (Hibbitt et al. Accepted).

A

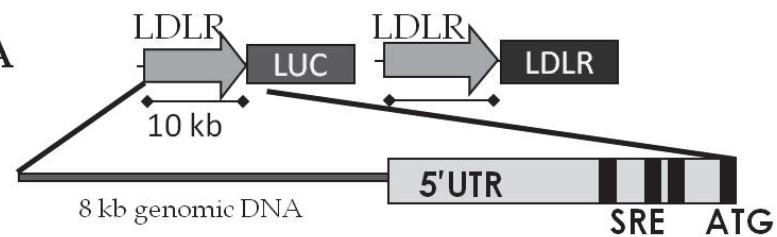

LDLR genomic promoter $2 \mathrm{~kb}$
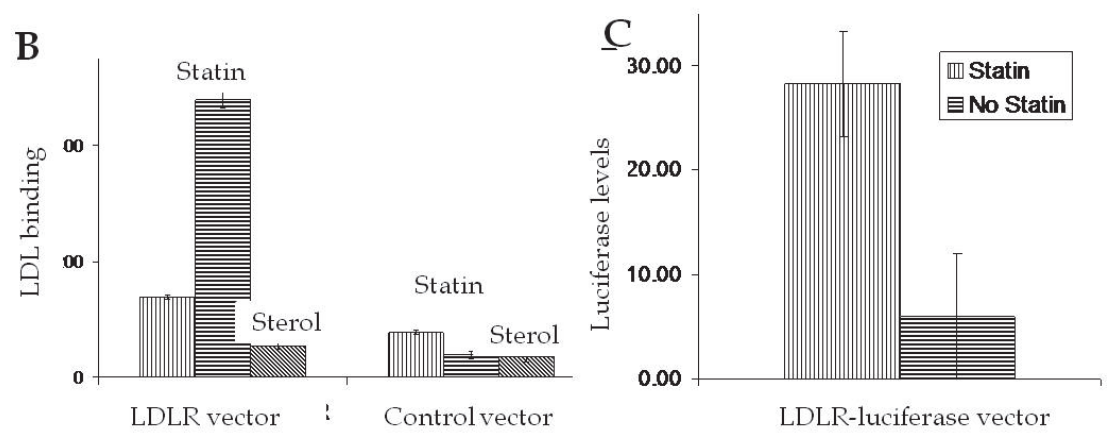

Generation of LDLR genomic mini-gene vectors. A) A 10kb Icece of genomic DNA encompassing the LDLR promoter the promoter region (UTR) and $8 \mathrm{~kb}$ upstream of genomic DNA was cloned into vectors containing the cDNA of either luciferase or LDLR. B) Following delivery of LDLR cDNA containing vectors to patient fibroblasts binding and internalisation of LDL was observed. Increased binding was seen after cells were treated with statins. Decreased binding was observed with cells treated with sterols. Cells transduced with a control vector that did not contain the LDLR expression cassette showed no binding and internalisation of LDL. C) Animals injected with luciferase expressing vectors and treated with statins show a 5 fold induction of LDLR promoter activity compared to animals no treated with statin. Results were obtained using live animal luciferase imaging. Figure modified from (Hibbitt et. al.,2010).

Fig. 3. Genomic mini-gene vectors

\section{Conclusion}

Gene replacement gene therapy has been under investigation for a number of years and is emerging as a potentially potent tool to treat genetic disease. Most gene therapy protocols involve the use of small cDNA vectors where expression of the transgene is constitutive and unregulated. While for some conditions this may be adequate, others will require the expression of therapeutic genes to be regulated spatially, temporally and physiologically to 
circumvent issues with genotoxicity, loss of expression, and lack of therapeutic effect in animal models.

Several advances have been made in recent years to address these issues. The use of transcriptional restriction is now wide-spread with many studies employing cell-specific promoters to ensure gene expression is limited to target cells. There have also been developments in the use of whole genomic DNA loci transgenes. This opens the possibility of using vectors for gene therapy which completely recapitulate endogenous expression. Advances in viral vectors based on helper virus-free HSV-1 amplicons mean that viral delivery of large genomic loci $>100 \mathrm{~kb}$ is now possible in vivo. Finally, the development of novel vectors which incorporate genomic DNA elements to achieve physiological expression in a mini-gene vector format will push the use of genomic regulatory elements in gene therapy vectors closer to a clinical reality.

A greater body of work in vivo is now needed using genomic regulatory vectors. Work is currently underway in our laboratory to assess the feasibility of using genomic mini-gene vectors to correct genetic defects in vivo long-term. We believe that these vectors represent a major addition to the gene therapy field and will be applicable to a wide range of genetic conditions.

\section{Acknowledgements}

Our work on gene therapy and vector development has been supported by the British Heart Foundation, the Medical Research Council, the Biotechnology and Biological Sciences Research Council, the Friedreich's Ataxia Research Alliance, and the Wellcome Trust.

\section{References}

Adrover, M. F., V. Guyot-Revol, et al. (2003). "Hippocampal infection with HSV-1-derived vectors expressing an NMDAR1 antisense modifies behavior." Genes Brain Behav 2(2): 103-113

Agudo, M., J. L. Trejo, et al. (2002). "Highly efficient and specific gene transfer to Purkinje cells in vivo using a herpes simplex virus I amplicon." Hum Gene Ther 13(5): 665-674

Aiuti, A., F. Cattaneo, et al. (2009). "Gene therapy for immunodeficiency due to adenosine deaminase deficiency." N Engl J Med 360(5): 447-458.1533-4406 (Electronic) 00284793 (Linking)

Bainbridge, J. W., A. J. Smith, et al. (2008). "Effect of gene therapy on visual function in Leber's congenital amaurosis." $N$ Engl J Med 358(21): 2231-2239.1533-4406 (Electronic) 0028-4793 (Linking)

Bochukova, E. G., A. Jefferson, et al. (2003). "Genomic studies of gene expression: regulation of the Wilson disease gene." Genomics 81(6): 531-542

Borst, E. and M. Messerle (2000). "Development of a cytomegalovirus vector for somatic gene therapy." Bone Marrow Transplant 25 Suppl 2: S80-82.0268-3369 (Print) 02683369 (Linking)

Borst, E. M. and M. Messerle (2003). "Construction of a cytomegalovirus-based amplicon: a vector with a unique transfer capacity." Hum Gene Ther 14(10): 959-970 
Bowers, W. J., J. A. Olschowka, et al. (2003). "Immune responses to replication-defective HSV-1 type vectors within the CNS: implications for gene therapy." Gene Ther 10(11): 941-945

Briggs, M. R., C. Yokoyama, et al. (1993). "Nuclear protein that binds sterol regulatory element of low density lipoprotein receptor promoter. I. Identification of the protein and delineation of its target nucleotide sequence." J Biol Chem 268(19): 14490-14496

Carvajal, J. J., D. Cox, et al. (2001). "A BAC transgenic analysis of the Mrf4/Myf5 locus reveals interdigitated elements that control activation and maintenance of gene expression during muscle development." Development 128(10): 1857-1868

Cavazzana-Calvo, M., S. Hacein-Bey, et al. (2000). "Gene therapy of human severe combined immunodeficiency (SCID)-X1 disease." Science 288(5466): 669-672.0036-8075 (Print) 0036-8075 (Linking)

Chi, X., S. X. Zhang, et al. (2003). "Expression of Nkx2-5-GFP bacterial artificial chromosome transgenic mice closely resembles endogenous Nkx2-5 gene activity." Genesis 35(4): 220-226

Chowdhury, J. R., M. Grossman, et al. (1991). "Long-term improvement of hypercholesterolemia after ex vivo gene therapy in LDLR-deficient rabbits." Science 254(5039): 1802-1805

Corey, L. and P. G. Spear (1986). "Infections with herpes simplex viruses (1)." N Engl J Med 314(11): 686-691

Cossee, M., H. Puccio, et al. (2000). "Inactivation of the Friedreich ataxia mouse gene leads to early embryonic lethality without iron accumulation." Hum Mol Genet 9(8): 12191226

Costantini, L. C., D. R. Jacoby, et al. (1999). "Gene transfer to the nigrostriatal system by hybrid herpes simplex virus/adeno-associated virus amplicon vectors." Hum Gene Ther 10(15): 2481-2494

Dupre, L., S. Trifari, et al. (2004). "Lentiviral vector-mediated gene transfer in T cells from Wiskott-Aldrich syndrome patients leads to functional correction." Mol Ther 10(5): 903-915.1525-0016 (Print) 1525-0016 (Linking)

Follenzi, A., G. Sabatino, et al. (2002). "Efficient gene delivery and targeted expression to hepatocytes in vivo by improved lentiviral vectors." Hum Gene Ther 13(2): 243260.1043-0342 (Print) 1043-0342 (Linking)

Frecha, C., M. G. Toscano, et al. (2008). "Improved lentiviral vectors for Wiskott-Aldrich syndrome gene therapy mimic endogenous expression profiles throughout haematopoiesis." Gene Ther 15(12): 930-941.1476-5462 (Electronic) 0969-7128 (Linking)

Gimenez-Cassina, A., R. Wade-Martins, et al. (2011). "Infectious delivery and long-term persistence of transgene expression in the brain by a 135-kb iBAC-FXN genomic DNA expression vector." Gene Ther.1476-5462 (Electronic) 0969-7128 (Linking)

Goss, J. R., W. F. Goins, et al. (2002). "Herpes simplex-mediated gene transfer of nerve growth factor protects against peripheral neuropathy in streptozotocin-induced diabetes in the mouse." Diabetes 51(7): 2227-2232 
Grossman, M., D. J. Rader, et al. (1995). "A pilot study of ex vivo gene therapy for homozygous familial hypercholesterolaemia." Nat Med 1(11): 1148-1154

Hibbitt, O., S. Agkatsev, et al. (Accepted). "RNAi-mediated knockdown of HMG CoA reductase enhances gene expression from physiologically-regulated low density lipoprotein receptor (LDLR) therapeutic vectors in vivo." Gene Ther

Hibbitt, O. C., R. P. Harbottle, et al. (2007). "Delivery and long-term expression of a $135 \mathrm{~kb}$ LDLR genomic DNA locus in vivo by hydrodynamic tail vein injection." J Gene Med 9(6): 488-497

Hibbitt, O. C., E. McNeil, et al. (2010). "Long-term physiologically regulated expression of the low-density lipoprotein receptor in vivo using genomic DNA mini-gene constructs." Mol Ther 18(2): 317-326.1525-0024 (Electronic) 1525-0016 (Linking)

Hibbitt, O. C. and R. Wade-Martins (2006). "Delivery of large genomic DNA inserts $>100 \mathrm{~kb}$ using HSV-1 amplicons." Curr Gene Ther 6(3): 325-336

Horton, J. D., J. L. Goldstein, et al. (2002). "SREBPs: activators of the complete program of cholesterol and fatty acid synthesis in the liver." J Clin Invest 109(9): 1125-1131

Hsiao, K., P. Chapman, et al. (1996). "Correlative memory deficits, Abeta elevation, and amyloid plaques in transgenic mice." Science 274(5284): 99-102

Huang, Y., D. P. Liu, et al. (2000). "Proper developmental control of human globin genes reproduced by transgenic mice containing a $160-\mathrm{kb}$ BAC carrying the human betaglobin locus." Blood Cells Mol Dis 26(6): 598-610

Imai, T., T. Morita, et al. (2001). "Vascular smooth muscle cell-directed overexpression of heme oxygenase- 1 elevates blood pressure through attenuation of nitric oxideinduced vasodilation in mice." Circ Res 89(1): 55-62.1524-4571 (Electronic) 0009-7330 (Linking)

Inoue, R., K. A. Moghaddam, et al. (2004). "Infectious delivery of the $132 \mathrm{~kb}$ CDKN2A/CDKN2B genomic DNA region results in correctly spliced gene expression and growth suppression in glioma cells." Gene Ther 11(15): 1195-1204

Ishibashi, S., M. S. Brown, et al. (1993). "Hypercholesterolemia in low density lipoprotein receptor knockout mice and its reversal by adenovirus-mediated gene delivery." $J$ Clin Invest 92(2): 883-893

Ishitobi, H., K. Matsumoto, et al. (2010). "Flk1-GFP BAC Tg mice: an animal model for the study of blood vessel development." Exp Anim 59(5): 615-622.1881-7122 (Electronic) 1341-1357 (Linking)

Jacobs, F., E. Van Craeyveld, et al. (2008). "Adenoviral low density lipoprotein receptor attenuates progression of atherosclerosis and decreases tissue cholesterol levels in a murine model of familial hypercholesterolemia." Atherosclerosis 201(2): 289297.1879-1484 (Electronic) 0021-9150 (Linking)

Jamsai, D., F. Zaibak, et al. (2005). "A humanized mouse model for a common beta0thalassemia mutation." Genomics 85(4): 453-461

Jamsai, D., F. Zaibak, et al. (2006). "A humanized BAC transgenic/knockout mouse model for $\mathrm{HbE}$ / beta-thalassemia." Genomics 88(3): 309-315

Kankkonen, H. M., E. Vahakangas, et al. (2004). "Long-term lowering of plasma cholesterol levels in LDL-receptor-deficient WHHL rabbits by gene therapy." Mol Ther 9(4): 548-556 
Kassim, S. H., H. Li, et al. (2010). "Gene therapy in a humanized mouse model of familial hypercholesterolemia leads to marked regression of atherosclerosis." PLoS One 5(10): e13424.1932-6203 (Electronic) 1932-6203 (Linking)

Kotzamanis, G., H. Abdulrazzak, et al. (2009). "CFTR expression from a BAC carrying the complete human gene and associated regulatory elements." J Cell Mol Med 13(9A): 2938-2948.1582-4934 (Electronic) 1582-1838 (Linking)

Kozarsky, K. F., K. Jooss, et al. (1996). "Effective treatment of familial hypercholesterolaemia in the mouse model using adenovirus-mediated transfer of the VLDL receptor gene." Nat Genet 13(1): 54-62

Kozarsky, K. F., D. R. McKinley, et al. (1994). "In vivo correction of low density lipoprotein receptor deficiency in the Watanabe heritable hyperlipidemic rabbit with recombinant adenoviruses." J Biol Chem 269(18): 13695-13702

Kulik, L., K. Chen, et al. (2011). "Human complement receptor type 2 (CR2/CD21) transgenic mice provide an in vivo model to study immunoregulatory effects of receptor antagonists." Mol Immunol 48(6-7): 883-894.1872-9142 (Electronic) 01615890 (Linking)

Lamb, B. T. (1995). "Making models for Alzheimer's disease." Nat Genet 9(1): 4-6

Lamb, B. T., L. M. Call, et al. (1997). "Altered metabolism of familial Alzheimer's diseaselinked amyloid precursor protein variants in yeast artificial chromosome transgenic mice." Hum Mol Genet 6(9): 1535-1541

Le, M., T. Okuyama, et al. (1997). "Therapeutic levels of functional human factor X in rats after retroviral-mediated hepatic gene therapy." Blood 89(4): 1254-1259.0006-4971 (Print) 0006-4971 (Linking)

Le Meur, G., K. Stieger, et al. (2007). "Restoration of vision in RPE65-deficient Briard dogs using an AAV serotype 4 vector that specifically targets the retinal pigmented epithelium." Gene Ther 14(4): 292-303.0969-7128 (Print) 0969-7128 (Linking)

Lebherz, C., G. Gao, et al. (2004). "Gene therapy with novel adeno-associated virus vectors substantially diminishes atherosclerosis in a murine model of familial hypercholesterolemia." J Gene Med 6(6): 663-672

Leuci, V., L. Gammaitoni, et al. (2009). "Efficient transcriptional targeting of human hematopoietic stem cells and blood cell lineages by lentiviral vectors containing the regulatory element of the Wiskott-Aldrich syndrome gene." Stem Cells 27(11): 28152823.1549-4918 (Electronic) 1066-5099 (Linking)

Li, J., B. Fang, et al. (1995). "In vivo gene therapy for hyperlipidemia: phenotypic correction in Watanabe rabbits by hepatic delivery of the rabbit LDL receptor gene." J Clin Invest 95(2): 768-773

Li, Y., W. Liu, et al. (2009). "Mutant LRRK2(R1441G) BAC transgenic mice recapitulate cardinal features of Parkinson's disease." Nat Neurosci 12(7): 826-828.1546-1726 (Electronic) 1097-6256 (Linking)

Lufino, M., O. Hibbitt, et al. (2007). Long-term regulated functional complementation of LDLR deficiency in cells using iBAC-SMAR-LDLR, a novel infectious genomic DNA episomal vector. American Society for Gene Therapy Annual Main Meeting, Seattle, Washington. 
Lufino, M. M., R. Manservigi, et al. (2007). "An S/MAR-based infectious episomal genomic DNA expression vector provides long-term regulated functional complementation of LDLR deficiency." Nucleic Acids Res 35(15): e98

Magin-Lachmann, C., G. Kotzamanis, et al. (2004). "In vitro and in vivo delivery of intact BAC DNA -- comparison of different methods." J Gene Med 6(2): 195-209

Magram, J., K. Chada, et al. (1985). "Developmental regulation of a cloned adult beta-globin gene in transgenic mice." Nature 315(6017): 338-340

Manno, C. S., G. F. Pierce, et al. (2006). "Successful transduction of liver in hemophilia by AAV-Factor IX and limitations imposed by the host immune response." Nat Med 12(3): 342-347.1078-8956 (Print) 1078-8956 (Linking)

Manson, A. L., A. E. Trezise, et al. (1997). "Complementation of null CF mice with a human CFTR YAC transgene." Embo J 16(14): 4238-4249

Marsh, D. R., G. A. Dekaban, et al. (2000). "Herpes simplex viral and amplicon vectormediated gene transfer into glia and neurons in organotypic spinal cord and dorsal root ganglion cultures." Mol Ther 1(5 Pt 1): 464-478

Martin, F., M. G. Toscano, et al. (2005). "Lentiviral vectors transcriptionally targeted to hematopoietic cells by WASP gene proximal promoter sequences." Gene Ther 12(8): 715-723.0969-7128 (Print) 0969-7128 (Linking)

May, C., S. Rivella, et al. (2000). "Therapeutic haemoglobin synthesis in beta-thalassaemic mice expressing lentivirus-encoded human beta-globin." Nature 406(6791): 82-86

Miranda, C. J., M. M. Santos, et al. (2002). "Frataxin knockin mouse." FEBS Lett 512(1-3): 291297

Miyanohara, A., M. F. Sharkey, et al. (1988). "Efficient expression of retroviral vectortransduced human low density lipoprotein (LDL) receptor in LDL receptordeficient rabbit fibroblasts in vitro." Proc Natl Acad Sci U S A 85(17): 6538-6542

Moralli, D., K. M. Simpson, et al. (2006). "A novel human artificial chromosome gene expression system using herpes simplex virus type 1 vectors." EMBO Rep 7(9): 911918.1469-221X (Print) 1469-221X (Linking)

Muller, L., O. Saydam, et al. (2005). "Gene transfer into hepatocytes mediated by herpes simplex virus-Epstein-Barr virus hybrid amplicons." J Virol Methods 123(1): 65-72

Nicklin, S. A., P. N. Reynolds, et al. (2001). "Analysis of cell-specific promoters for viral gene therapy targeted at the vascular endothelium." Hypertension 38(1): 65-70.1524-4563 (Electronic) 0194-911X (Linking)

Nomura, S., A. Merched, et al. (2004). "Low-density lipoprotein receptor gene therapy using helper-dependent adenovirus produces long-term protection against atherosclerosis in a mouse model of familial hypercholesterolemia." Gene Ther 11(20): 1540-1548

Olschowka, J. A., W. J. Bowers, et al. (2003). "Helper-free HSV-1 amplicons elicit a markedly less robust innate immune response in the CNS." Mol Ther 7(2): 218-227

Ott, M. G., M. Schmidt, et al. (2006). "Correction of X-linked chronic granulomatous disease by gene therapy, augmented by insertional activation of MDS1-EVI1, PRDM16 or SETBP1." Nat Med 12(4): 401-409.1078-8956 (Print) 1078-8956 (Linking) 
Peruzzi, P. P., S. E. Lawler, et al. (2009). "Physiological transgene regulation and functional complementation of a neurological disease gene deficiency in neurons." Mol Ther 17(9): 1517-1526.1525-0024 (Electronic) 1525-0016 (Linking)

Porcu, S., M. Kitamura, et al. (1997). "The human beta globin locus introduced by YAC transfer exhibits a specific and reproducible pattern of developmental regulation in transgenic mice." Blood 90(11): 4602-4609

Pritchard, L., J. A. Sloane-Stanley, et al. (2000). "A human PKD1 transgene generates functional polycystin- 1 in mice and is associated with a cystic phenotype." Hum Mol Genet 9(18): 2617-2627

Quinn, G., T. Ochiya, et al. (2000). "Mouse flt-1 promoter directs endothelial-specific expression in the embyroid body model of embryogenesis." Biochem Biophys Res Commun 276(3): 1089-1099.0006-291X (Print) 0006-291X (Linking)

Rocchi, L., C. Braz, et al. (2010). "Escherichia coli-cloned CFTR loci relevant for human artificial chromosome therapy." Hum Gene Ther 21(9): 1077-1092.1557-7422 (Electronic) 1043-0342 (Linking)

Saeki, Y., X. O. Breakefield, et al. (2003). "Improved HSV-1 amplicon packaging system using ICP27-deleted, oversized HSV-1 BAC DNA." Methods Mol Med 76: $51-60$

Saeki, Y., T. Ichikawa, et al. (1998). "Herpes simplex virus type 1 DNA amplified as bacterial artificial chromosome in Escherichia coli: rescue of replicationcompetent virus progeny and packaging of amplicon vectors." Hum Gene Ther 9(18): 2787-2794

Sarsero, J. P., L. Li, et al. (2004). "Human BAC-mediated rescue of the Friedreich ataxia knockout mutation in transgenic mice." Mamm Genome 15(5): 370-382

Schiedner, G., N. Morral, et al. (1998). "Genomic DNA transfer with a high-capacity adenovirus vector results in improved in vivo gene expression and decreased toxicity." Nat Genet 18(2): 180-183

Senior, S. L. and R. Wade-Martins (2005). "Herpes simplex virus type 1 amplicon vectors for the infectious delivery and expression of genomic DNA loci." Curr Opin Mol Ther 7(4): 337-345

Shah, K., C. H. Tung, et al. (2004). "In vivo imaging of HIV protease activity in amplicon vector-transduced gliomas." Cancer Res 64(1): 273-278

Sharpless, N. E. and R. A. DePinho (1999). "The INK4A/ARF locus and its two gene products." Curr Opin Genet Dev 9(1): 22-30

Spaete, R. R. and N. Frenkel (1982). "The herpes simplex virus amplicon: a new eucaryotic defective-virus cloning-amplifying vector." Cell 30(1): 295-304

Spaete, R. R. and N. Frenkel (1985). "The herpes simplex virus amplicon: analyses of cisacting replication functions." Proc Natl Acad Sci U S A 82(3): 694-698

Stavropoulos, T. A. and C. A. Strathdee (1998). "An enhanced packaging system for helperdependent herpes simplex virus vectors." J Virol 72(9): 7137-7143

Sudhof, T. C., D. W. Russell, et al. (1987). "42 bp element from LDL receptor gene confers end-product repression by sterols when inserted into viral TK promoter." Cell 48(6): 1061-1069 
Thies, R. S., M. Bauduy, et al. (1992). "Recombinant human bone morphogenetic protein-2 induces osteoblastic differentiation in W-20-17 stromal cells." Endocrinology 130(3): 1318-1324

Toscano, M. G., C. Frecha, et al. (2008). "Hematopoietic-specific lentiviral vectors circumvent cellular toxicity due to ectopic expression of Wiskott-Aldrich syndrome protein." Hum Gene Ther 19(2): 179-197.1043-0342 (Print) 1043-0342 (Linking)

Vadolas, J., H. Wardan, et al. (2005). "Transgene copy number-dependent rescue of murine beta-globin knockout mice carrying a $183 \mathrm{~kb}$ human beta-globin BAC genomic fragment." Biochim Biophys Acta 1728(3): 150-162

Vadolas, J., H. Wardan, et al. (2002). "Development of sensitive fluorescent assays for embryonic and fetal hemoglobin inducers using the human beta -globin locus in erythropoietic cells." Blood 100(12): 4209-4216

Van Craeyveld, E., S. C. Gordts, et al. (2011). "Regression and stabilization of advanced murine atherosclerotic lesions: a comparison of LDL lowering and HDL raising gene transfer strategies." J Mol Med.1432-1440 (Electronic) 09462716 (Linking)

Wade-Martins, R., Y. Saeki, et al. (2003). "Infectious delivery of a 135-kb LDLR genomic locus leads to regulated complementation of low-density lipoprotein receptor deficiency in human cells." Mol Ther 7(5 Pt 1): 604-612

Wade-Martins, R., E. R. Smith, et al. (2001). "An infectious transfer and expression system for genomic DNA loci in human and mouse cells." Nat Biotechnol 19(11): 1067-1070

Wade-Martins, R., R. E. White, et al. (2000). "Stable correction of a genetic deficiency in human cells by an episome carrying a $115 \mathrm{~kb}$ genomic transgene." Nat Biotechnol 18(12): 1311-1314.1087-0156 (Print) 1087-0156 (Linking)

Wang, S. and J. M. Vos (1996). "A hybrid herpesvirus infectious vector based on Epstein-Barr virus and herpes simplex virus type 1 for gene transfer into human cells in vitro and in vivo." J Virol 70(12): 8422-8430

Wang, Y., S. M. Camp, et al. (2002). "Herpes simplex virus type 1/adeno-associated virus rep $(+)$ hybrid amplicon vector improves the stability of transgene expression in human cells by site-specific integration." J Virol 76(14): 7150-7162

Wang, Y., C. Fraefel, et al. (2000). "HSV-1 amplicon vectors are a highly efficient gene delivery system for skeletal muscle myoblasts and myotubes." Am J Physiol Cell Physiol 278(3): C619-626

White, R. E., R. Wade-Martins, et al. (2003). "Functional delivery of large genomic DNA to human cells with a peptide-lipid vector." J Gene Med 5(10): 883-892

White, R. E., R. Wade-Martins, et al. (2002). "Infectious delivery of 120-kilobase genomic DNA by an epstein-barr virus amplicon vector." Mol Ther 5(4): 427-435

Wilson, J. M., D. E. Johnston, et al. (1988). "Correction of the genetic defect in hepatocytes from the Watanabe heritable hyperlipidemic rabbit." Proc Natl Acad Sci U S A 85(12): 4421-4425

Xing, W., D. Baylink, et al. (2004). "HSV-1 amplicon-mediated transfer of 128-kb BMP-2 genomic locus stimulates osteoblast differentiation in vitro." Biochem Biophys Res Commun 319(3): 781-786 
Zammit, P. S., J. J. Carvajal, et al. (2004). "Myf5 expression in satellite cells and spindles in adult muscle is controlled by separate genetic elements." Dev Biol 273(2): 454465

Zhou, L., C. R. Dey, et al. (1994). "Correction of lethal intestinal defect in a mouse model of cystic fibrosis by human CFTR." Science 266(5191): 1705-1708 


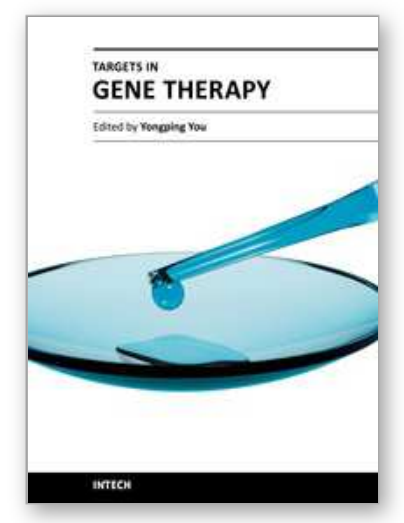

\author{
Targets in Gene Therapy \\ Edited by Prof. Yongping You
}

ISBN 978-953-307-540-2

Hard cover, 436 pages

Publisher InTech

Published online 23, August, 2011

Published in print edition August, 2011

This book aims at providing an up-to-date report to cover key aspects of existing problems in the emerging field of targets in gene therapy. With the contributions in various disciplines of gene therapy, the book brings together major approaches: Target Strategy in Gene Therapy, Gene Therapy of Cancer and Gene Therapy of Other Diseases. This source enables clinicians and researchers to select and effectively utilize new translational approaches in gene therapy and analyze the developments in target strategy in gene therapy.

\title{
How to reference
}

In order to correctly reference this scholarly work, feel free to copy and paste the following:

Olivia Hibbitt and Richard Wade-Martins (2011). Physiologically-Regulated Expression Vectors for Gene Therapy, Targets in Gene Therapy, Prof. Yongping You (Ed.), ISBN: 978-953-307-540-2, InTech, Available from: http://www.intechopen.com/books/targets-in-gene-therapy/physiologically-regulated-expression-vectorsfor-gene-therapy

\section{INTECH}

open science | open minds

\author{
InTech Europe \\ University Campus STeP Ri \\ Slavka Krautzeka 83/A \\ 51000 Rijeka, Croatia \\ Phone: +385 (51) 770447 \\ Fax: +385 (51) 686166 \\ www.intechopen.com
}

\author{
InTech China \\ Unit 405, Office Block, Hotel Equatorial Shanghai \\ No.65, Yan An Road (West), Shanghai, 200040, China \\ 中国上海市延安西路65号上海国际贵都大饭店办公楼 405 单元 \\ Phone: +86-21-62489820 \\ Fax: $+86-21-62489821$
}


(C) 2011 The Author(s). Licensee IntechOpen. This chapter is distributed under the terms of the Creative Commons Attribution-NonCommercialShareAlike-3.0 License, which permits use, distribution and reproduction for non-commercial purposes, provided the original is properly cited and derivative works building on this content are distributed under the same license. 